\title{
Microstructure Control of Columnar-Grained Silicon Substrate Solidified from Silicon Melts Using Gas Pressure
}

\author{
Jun-Kyu Lee, ${ }^{1,2}$ Sung-Min Wi, ${ }^{1}$ Jin-Seok Lee, ${ }^{1}$ Bo-Yun Jang, ${ }^{1}$ Joon-Soo Kim, \\ Young-Soo Ahn, ${ }^{1}$ and Churl-Hee $\mathrm{Cho}^{2}$ \\ ${ }^{1}$ Advanced Materials and Devices Laboratory, Korea Institute of Energy Research, Daejeon 305-343, Republic of Korea \\ ${ }^{2}$ Graduate School of Energy Science and Technology, Chungnam National University, Daejeon 305-764, Republic of Korea \\ Correspondence should be addressed to Jin-Seok Lee; jslee@kier.re.kr and Churl-Hee Cho; choch@cnu.ac.kr
}

Received 17 April 2015; Revised 24 August 2015; Accepted 25 August 2015

Academic Editor: Stefano Bellucci

Copyright (C) 2015 Jun-Kyu Lee et al. This is an open access article distributed under the Creative Commons Attribution License, which permits unrestricted use, distribution, and reproduction in any medium, provided the original work is properly cited.

A silicon substrate with the dimensions of $100 \times 140 \times 0.3 \mathrm{~mm}$ was grown directly from liquid silicon with gas pressure. The silicon melt in the sealed melting part was injected into the growth part at applied pressure of 780-850 Torr. The solidified silicon substrate was then transferred by the pull of the cooled dummy bar. A desirable structure with a liquid-solid interface perpendicular to the pulling direction was formed when the mold temperature in the solidification zone of the growth part was much higher than that of the dummy bar, as this technique should be able to overcome thermal loss through the molds and the limited heat flux derived from the very narrow contact area between the silicon melt and the dummy bar. In addition, because the metallic impurities and expansion of volume during solidification are preferably moved to a liquid phase, a high-quality silicon substrate, without defects such as cracks and impurities in the substrate, could be manufactured in the interface structure. The present study reports the experimental findings on a new and direct growth system for obtaining silicon substrates characterized by high quality and productivity, as a candidate for alternate routes for the fabrication of silicon substrates.

\section{Introduction}

Silicon ( $\mathrm{Si}$ ) is one of the important fundamental materials in the modern semiconductor industry, which could be prepared by chemical and metallurgical route as well as recycling of end-of-life photovoltaic module. It is also very useful as an electronic substrate material $[1,2]$. The most conventional method of fabricating Si substrate consists in slicing Si bricks. However, this method has some economic disadvantages resulting from kerf-loss and the limited size of the substrate thus produced [3]. The recent tendency in $\mathrm{Si}$ substrate technology has been towards improving $\mathrm{Si}$ utilization, such as minimizing kerf-loss, when the ingots are cut off. The most available substrates have been manufactured by sawing Si bricks, which are grown by various techniques including the Czochralski and Bridgman methods. Kerf-loss of around $50 \%$ is generated during the slicing process of a single crystalline ingot or polycrystalline block. In order to resolve this problem, active studies are being carried out on a technique for directly manufacturing a $\mathrm{Si}$ substrate from molten $\mathrm{Si}$ without the ingot slicing step [4]. These techniques are classified according to the transport direction of the solidified substrate with respect to the movement of the liquid-solid interface during crystallization. Vertical growth methods such as edge-defied film-fed growth (EFG) and string ribbon (SR) growth have a liquid-solid interface moving in a line perpendicular to the substrate transport direction [5-7]. Conversely, horizontal growth methods such as ribbon growth on substrate (RGS) and crystallization on dipped substrate (CDS) have a liquid-solid interface moving almost parallel to the substrate transport direction $[7,8]$. Even though the growth rate of EFG and SR is very slow at just 1$2 \mathrm{~cm} / \mathrm{min}$, products derived from the vertical growth method have already been commercialized due to their excellent electronics performance with large grain sizes. On the other hand, products obtained by horizontal growth methods such as RGS and CDS have restricted market entry despite their very fast growth rate of around $600 \mathrm{~cm} / \mathrm{min}$, because 


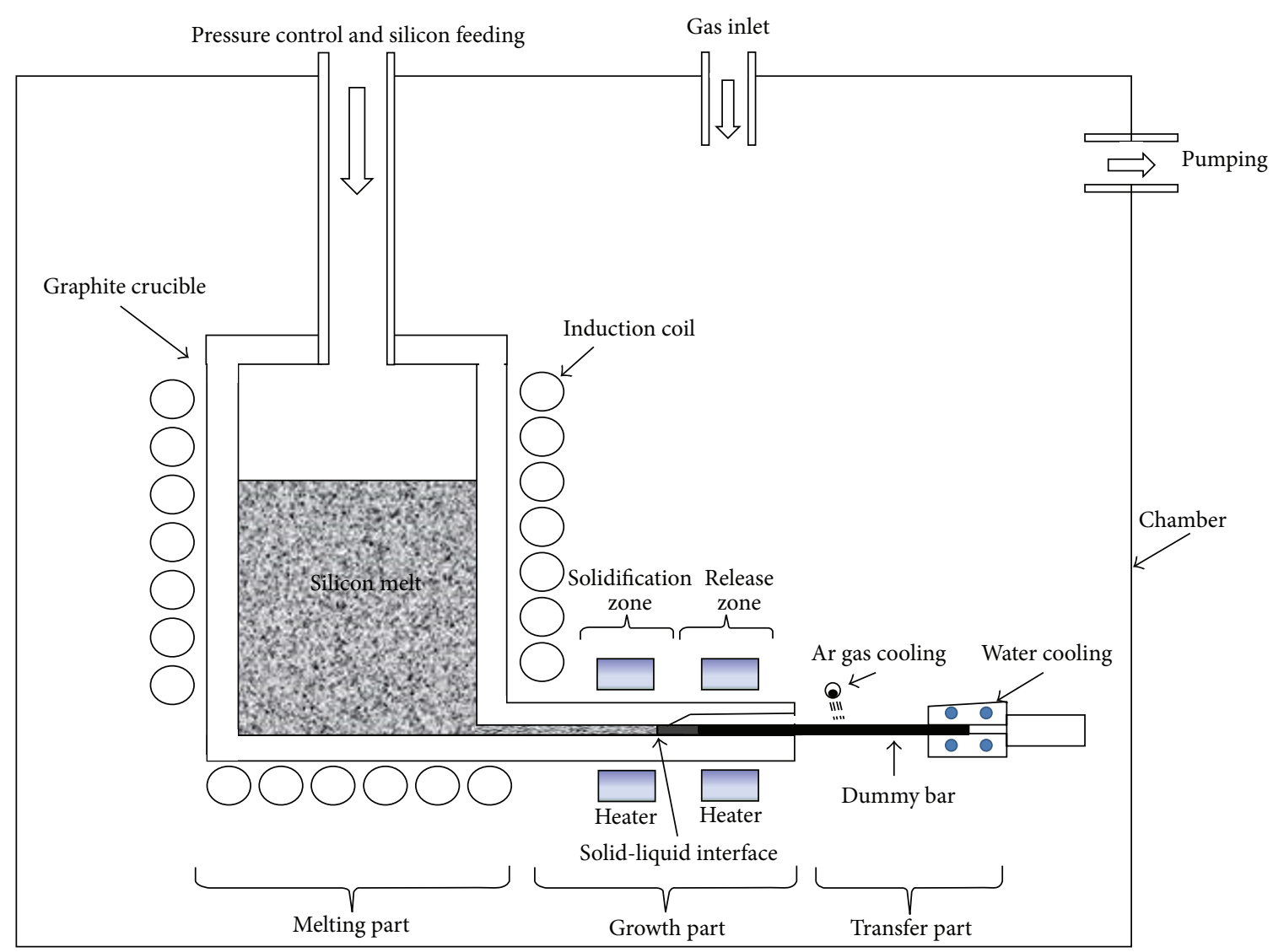

FIGURE 1: Schematic diagram of the novel growth system for directly manufacturing Si substrate.

the possibility of improving their electronics performance is extremely limited due to the very small grain size of around $100 \mu \mathrm{m}$ [9]. Due to this concern, a novel technique for simultaneously achieving a fast growth rate and large grains in the growth technology for Si substrate is necessary. The present investigation reports a new direct growth system for obtaining Si substrate characterized by high quality and productivity from Si melts under gas pressure by controlling the shape of the liquid-solid interface.

\section{Experimental Procedure}

A novel direct growth system for $\mathrm{Si}$ substrate was set up, as shown in Figure 1. The system consisted of a sealed part for the melting of Si feedstock, a growth part for $\mathrm{Si}$ substrates of a desired dimension, and a substrate transfer part for continuous growth by a dummy bar in a vacuum chamber, in which all three parts were made with SiCcoated graphite materials offering high thermal conductivity and chemical resistance. In particular, the growth part was designed specifically to compensate the expansion of $\mathrm{Si}$ volume under phase transformation. The pressure of the sealed melting part can be independently controlled by using the pressure of the main chamber. After perfectly melting the Si feedstock of extremely high purity (99.9999999\%) in the melting part using applied RF induction power at a chamber pressure of $10^{-2}$ Torr, the pressure of the chamber was attained at 760 Torr by adjusting the Ar gas. Then, Ar gas was applied to increase the pressure in the sealed melting part up to 850 Torr on the surface of the Si melt in order to inject the melts into the growth part positioned on the side of the melting part. The dimensions of the substrate were determined by the space in the growth zones. The growth part consisted of a solidification zone and a release zone, with the solidification zone designed to verify the temperature at a range of $1400-1600^{\circ} \mathrm{C}$, although the temperature of the release zone was fixed at $1100^{\circ} \mathrm{C}$ using SiC-coated graphite heaters. The solidification of liquid Si was initiated via contact with a cooled and porous dummy bar. Finally, the solidified Si substrate was continuously transferred outside the growth part by pulling, using the dummy bar at a pulling speed of $60 \mathrm{~cm} / \mathrm{min}$. The surface and cross section of the Si substrates were etched using a $45 \% \mathrm{KOH}$ solution, and microstructure observations for the Si substrates were carried out using an optical microscope (BX60MF, Olympus, Japan). The average grain sizes were calculated according to ASTM standard (E112-12) [10].

\section{Results and Discussion}

Figure 2 shows the Si substrate measuring $100 \times 140 \times 0.3 \mathrm{~mm}$ that was successfully grown in the novel manufacturing 


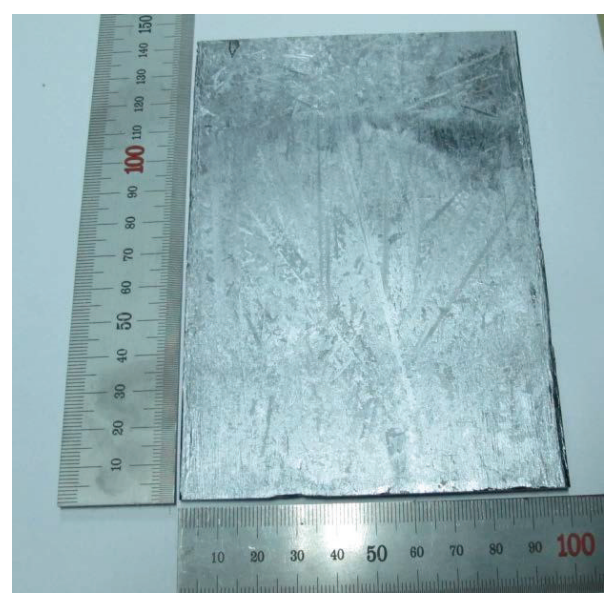

(a)

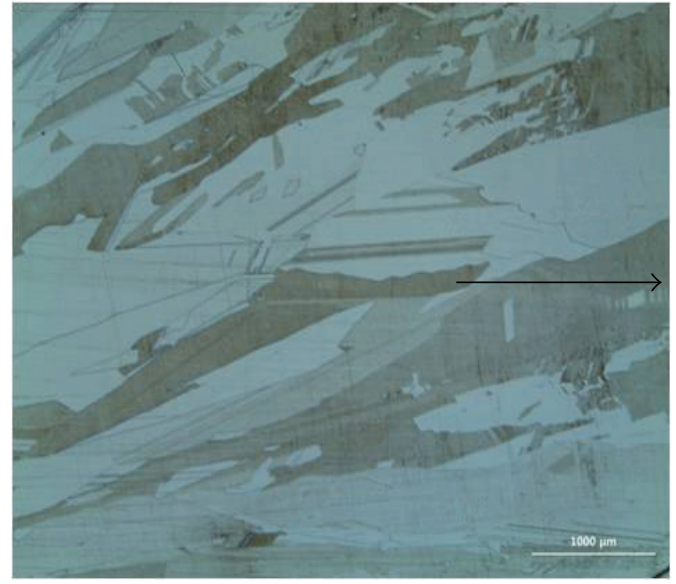

(b)

Figure 2: (a) Photograph of as-grown Si substrate and (b) optical microstructure on the surface of the substrate. The arrow indicates the pulling direction.

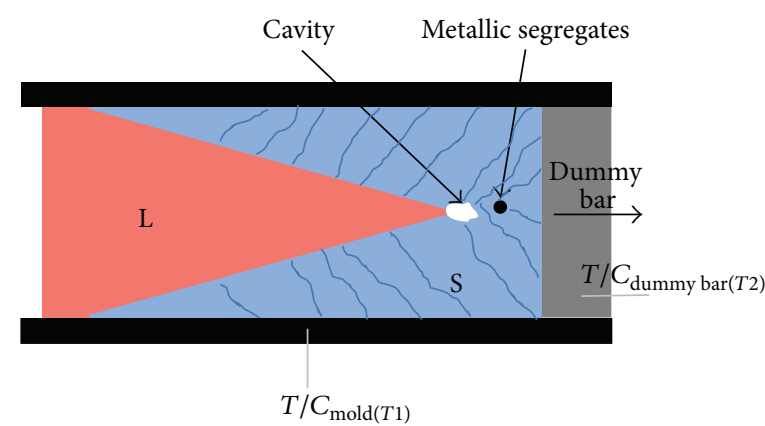

(a)

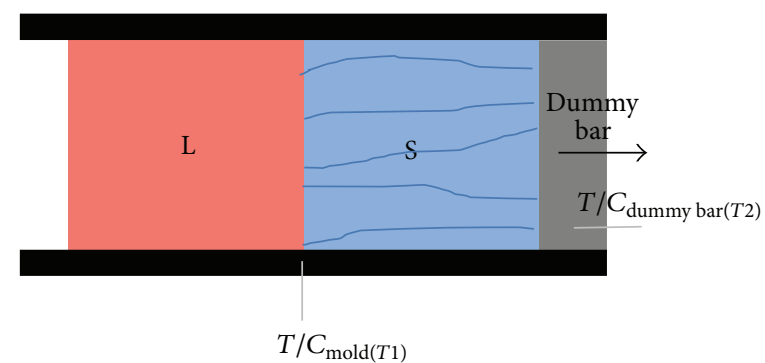

(b)

FIGURE 3: Schematic diagram of the structural formations of the liquid-solid interface depending on the difference between the temperature of the mold (T1) and the temperature of the dummy bar (T2) in the solidification zone of growth part: (a) $T 1 \leq T 2$ and (b) $T 1 \gg T 2$. The arrows indicate the pulling directions. $\mathrm{L}$ and $\mathrm{S}$ represent liquids and solids, respectively.

equipment with its microstructure positioned on the surface of the substrate. The surface morphology of the Si substrate was relatively smooth due to growth in the extremely restricted space in the growth part. The average size of the grains of the Si substrate grown at a mold temperature of $1600^{\circ} \mathrm{C}$ was calculated based on the ASTM standard [10], so that it presented the large grain size of $1.5 \mathrm{~mm}^{2}$ greater than $1 \mathrm{~mm}$ [9] with a high aspect ratio. One of the most important elements of this technique is the pressure control of Ar gas on the melt surface in the sealed melting part. Even though the substrate growth part was set at an atmospheric pressure that was equal to the chamber pressure, a low pressure of below 780 Torr in the sealed melting part could not inject the $\mathrm{Si}$ melts into the substrate growth part due to the very high surface tension $(>700 \mathrm{mN} / \mathrm{m})$ of the liquid $\mathrm{Si}$ in the crucible [11]. Pressure of at least 780 Torr was required in the sealed crucible to continuously fabricate the Si substrate. On the other hand, the melt flow could not be easily controlled in the case of very high pressure superior to 850 Torr due to the low viscosity $(0.55 \mathrm{mPa} \cdot \mathrm{s}$ at $1700 \mathrm{~K})$ of molten $\mathrm{Si}$ [11]. The microstructure on the surface of the substrate inferred that the grain growth of the Si progressed along the pulling direction, which meant that the liquid-solid interface seemed to move perpendicular to the pulling direction.

The key role of the dummy bar in this system is to remove the latent heat of molten Si during solidification toward the cooled dummy bar, thereby causing the liquid-solid interface to become perpendicular to the pulling direction. The interface structure, comprising the EFG and SR, is useful in compensating the expansion of volume generated during liquid-solid transformation and in purifying the substrate, because the volume expansion and impurities preferentially move to the liquid phase. Figure 3 shows a schematic diagram of the structural formation of the liquid-solid interface depending on the temperature difference between the mold and the dummy bar in the solidification zone of the growth part. $T 1$ and $T 2$ indicate the temperature of the SiC-coated graphite mold and the temperature of the cooled dummy bar, respectively. As shown in Figure 3(a), when the temperature of $T 1$ was either the same as $T 2$ or lower, the liquidsolid interface was formed relatively parallel to the pulling direction, similar to RGS and CDS. Therefore, cracks were 
formed inside the Si substrate due to the expansion of volume occasioned by the liquid-solid transformation, as solidification was completed with the substrate inside.

The metallic impurities can be induced by contamination in the silicon melting from physical contact of the graphite mold. The impurities in molten Si could be concentrated and trapped at the substrate. However, the concentration of impurities in the remaining liquid fraction becomes enriched during solidification, with the result that the solidified portion in the molten Si tends to be purified. This phenomenon can be quantified by the introduction of a segregation coefficient $k_{i}$ for each impurity $i$ defined as [12]

$$
k_{i}=\frac{C_{i}^{S}}{C_{i}^{L}}
$$

where $C_{i}^{S}$ is the concentration of impurities in the solid Si and $C_{i}^{L}$ is the concentration of impurities in the liquid Si. By a simple mass-balance analysis of the directional solidification process, it can be shown that the impurity profile in the $\mathrm{Si}$ substrate has the form after the solidification step [13]:

$$
C=C_{0} \cdot k \cdot(1-g)^{k-1},
$$

where $C$ is the concentration of impurities in the solidified $\mathrm{Si}, \mathrm{C}_{0}$ is the concentration of impurities in the charged $\mathrm{Si}, k$ is the segregation coefficient, and $g$ is the fraction solidified. The purification effect obtained by the preferential segregation of impurities can be quite dramatic since many of the metallic impurities in Si have segregation coefficients ranging from $10^{-5}$ to $10^{-1}$ [12]. Consequently, the structure of the liquidsolid interface, being parallel to the pulling direction, as shown in Figure 3(a), should be avoided for purification of the $\mathrm{Si}$ substrate, as the remaining liquid fraction with metallic segregates is finally solidified inside the substrate.

As regards the high extraction of latent heat through the cooled dummy bar, however, the liquid-solid interface was formed perpendicular to the pulling direction, similar to EFG and SR in the vertical growth system, as shown in Figure 3(b). This was due to the fact that the temperature of T1 was much higher than that of T2. However, a very slow growth rate and temperature control were needed, because most of the crystallization heat should be transported by a temperature gradient through the cooled dummy bar. This means that the crystal growth rate is controlled by the heat flux through the substrate by the dummy bar. In this regard, the equation for the calculation of the pulling velocity, $v_{p}$, being frequently used in growth systems with a liquidsolid interface perpendicular to the pulling direction, that is, similar to the vertical growth system, could be useful, as follows [14]:

$$
v_{p}=\frac{1}{L \rho_{m}}\left(\frac{\sigma \mathcal{\varepsilon}(W+t) K_{m} T_{m}^{5}}{W t}\right)^{1 / 2}
$$

where $L$ is the latent heat of fusion, $\rho_{m}$ is the density of the crystal at melting temperature, $\sigma$ is the Stefan-Boltzmann constant, $\varepsilon$ is the emissivity of the crystal, $t$ is the substrate thickness, $K_{m}$ is the thermal conductivity of the solid crystal at the melting temperature, $T_{m}$, and $W$ is the substrate width. For a $300 \mu \mathrm{m}$ thick substrate, (3) presents a maximum growth rate of $\sim 8 \mathrm{~cm} / \mathrm{min}$. However, typical growth rates are much lower due to the maximum tolerable thermal stress limiting the maximum tolerable temperature gradient in the substrate. The temperature gradient in the Si substrate is around $1000 \mathrm{~K} / \mathrm{cm}$ near the liquid-solid interface [15]. Therefore, a fast growth rate cannot be expected using the vertical growth system. However, the thermal stress was low in our novel direct growth technique. The reason for this occurrence was that once the $\mathrm{Si}$ substrate had been fabricated by solidification with the cooled dummy bar in the solidification zone, the remaining heat of the Si substrate was effectively and rapidly removed via the conduction mechanism through the molds of the release zone at the growth part. Therefore, this technique could achieve both a desirable microstructure and a higher growth rate.

In order to simultaneously derive a high growth rate and a large grain size from the desirable liquid-solid interface structure, the latent heat of the Si should be quickly transported to the dummy bar, thereby preventing the movement of the heat through the molds in the solidification zone. Molten Si should be solidified by conduction at the contact area between the molten Si and the dummy bar, where the dummy bar plays the role of a seed. The temperature of the dummy bar should be much lower than that of the liquid Si, because a lot of latent heat from the melts could not actually be transported toward the dummy bar due to the very narrow contact area $\left(30 \mathrm{~mm}^{2}\right)$ of the melt and the dummy bar. In order to overcome this limited geometrical factor, the heat flux rate of Si should be considered, using the following relation [16]:

$$
q=\rho C_{p} S \frac{d T}{d t}
$$

where $q$ is the heat flux in $\mathrm{Si}, \rho$ is the density, $C_{p}$ is the specific heat, $t$ is the time, $T$ is the temperature, and $S$ is the thickness of the dummy bar. According to (4), the heat flux increases as the thickness of the dummy bar, $S$, and temperature gradient, $\Delta T$, increases. In this system, the only valuable processing parameter was the temperature gradient, as the thickness of the dummy bar was fixed at $300 \mu \mathrm{m}$. Therefore, the key issue in this technique was how to lower the temperature of the dummy bar. The blowing system of Ar gas on the dummy bar was additionally introduced in order to reduce the temperature of the dummy bar, as there was a limitation with the cooling efficiency of a dummy bar with only a water cooling jacket.

Figure 4 shows cross section images of Si substrates as a function of mold temperature in the solidification zone of the growth part. Defect lines were clearly observed at the center of the substrate, as shown in Figures 4(a) and 4(b), which indicates that nucleation occurred at the surfaces of the upper/lower molds and that growth then proceeded toward the substrate inside. Cracks derived from volume expansion during solidification were observed even at the mold temperature of $1500^{\circ} \mathrm{C}$, which was higher than the melting temperature of $\mathrm{Si}$. This was due to the fact that the temperature measured by the thermocouple at the local position of the mold could differ somewhat from the temperature 


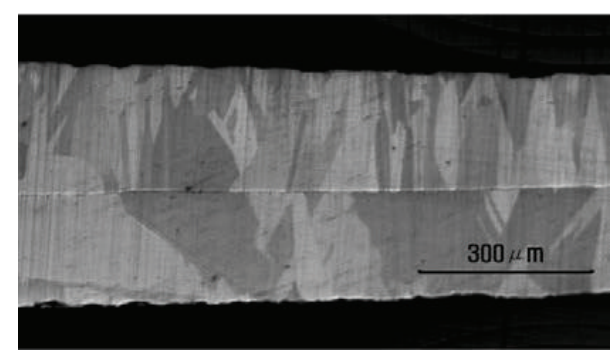

(a)

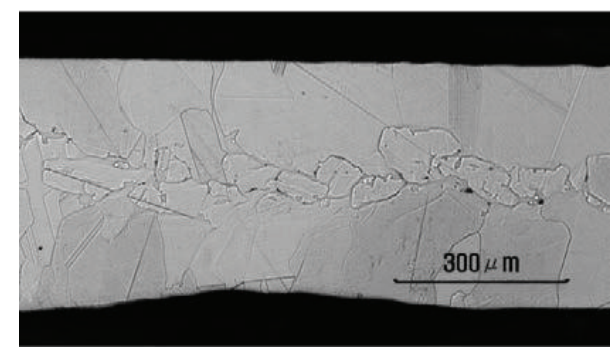

(b)

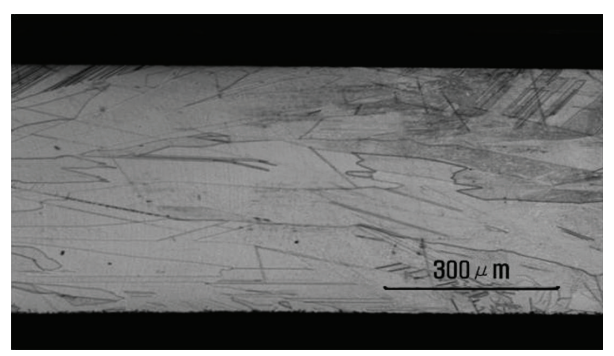

(c)

Figure 4: Cross section images of Si substrates as a function of mold temperature in the solidification zone of the growth part: at (a) $1400^{\circ} \mathrm{C}$, (b) $1500^{\circ} \mathrm{C}$, and (c) $1600^{\circ} \mathrm{C}$.

of the whole mold, which means that there was thermal loss through the molds. The temperatures of the molds were much higher than those of the dummy bars in both samples. Nevertheless, the latent heat of liquid Si was not effectively removed through the dummy bar, because of the very narrow contact area between the molten Si and the dummy bar. On the other hand, columnar grains were aligned to be parallel to the pulling direction in the case of a mold temperature of $1600^{\circ} \mathrm{C}$, similar to EFG and SR, as shown in Figure $4(\mathrm{c})$. This means that the liquid-solid interface moves almost perpendicular to the pulling direction, which indicates that the latent heat of $\mathrm{Si}$ was eliminated through the dummy bar. Consequently, these experimental findings demonstrate that the mold temperature should be much higher than the melting temperature of Si to overcome thermal loss through the mold and the limited heat flux derived from the very narrow contact area with the dummy bar.

\section{Conclusion}

In order to fabricate a Si substrate without kerf-loss, a novel direct system of growing $\mathrm{Si}$ substrate from Si melts using gas pressure was developed in order to satisfy demands for high quality and productivity. The system consisted of a sealed part for melting Si feedstock, a growth part for $\mathrm{Si}$ substrate of the desired dimensions, and a substrate transfer part for securing continuous growth using the dummy bar in a vacuum chamber. The gas pressure on the surface of the $\mathrm{Si}$ melts in the melting part should be in a range of 780-850 Torr when injecting the melt into the growth part, due to the very high surface tension and low viscosity of the silicon melts. In the case of a mold temperature of $\leq 1500^{\circ} \mathrm{C}$, defects such as cracks and impurities were generated at the center of the substrate by the formation of a liquid-solid interface structure running parallel to the pulling direction, such as RGS and CDS, which indicated that nucleation did not occur on the surface of the dummy bar due to thermal loss through the molds and the restricted heat flux toward the dummy bar. On the other hand, a desirable liquid-solid interface structure that was perpendicular to the pulling direction, such as EFG and SR, was formed at the mold temperature of $1600^{\circ} \mathrm{C}$ in the solidification zone of the growth part, because thermal loss and limited heat flux were overcome at that temperature. Consequently, the temperature of the mold should be much higher than the melting temperature of Si to form a liquid-solid interface structure perpendicular to the pulling direction, with nucleation occurring at the surface of the dummy bar, which was useful in compensating the volume expansion generated during liquid-solid transformation and in purifying the substrate, as the volume expansion and impurities preferentially move into the liquid phase.

\section{Conflict of Interests}

The authors declare that there is no conflict of interests regarding the publication of this paper.

\section{Acknowledgments}

This work was supported by the Research and Development Program of the Korea Institute of Energy Research (KIER) (B5-2464) and by the New \& Renewable Energy Technology Development Program of the Korea Institute of Energy Technology Evaluation and Planning (KETEP) with a grant 
funded by the Korean Government Ministry of Knowledge Economy (no. 20103020010060).

\section{References}

[1] G. H. Lee, C. K. Rhee, and K. S. Lim, "A study on the fabrication of polycrystalline Si wafer by direct casting for solar cell substrate," Solar Energy, vol. 80, no. 2, pp. 220-225, 2006.

[2] E. Menard, K. J. Lee, D.-Y. Khang, R. G. Nuzzo, and J. A. Rogers, "A printable form of silicon for high performance thin film transistors on plastic substrates," Applied Physics Letters, vol. 84, no. 26, pp. 5398-5400, 2004.

[3] G. G. Dongre, C. Vesvikar, R. K. Singh, and S. S. Joshi, "Efficient dicing of silicon ingots for photovoltaic applications," in Proceedings of the 35th IEEE Photovoltaic Specialists Conference (PVSC '10), pp. 3629-3634, IEEE, Honolulu, Hawaii, USA, June 2010.

[4] M. Suzuki, I. Hide, T. Yokoyama, T. Matsuyama, Y. Hatanaka, and Y. Maeda, "Growth of polycrystalline silicon sheet by Hoxan cast ribbon process," Journal of Crystal Growth, vol. 104, no. 1, pp. 102-107, 1990.

[5] J. P. Kalejs, B. H. Mackintosh, and T. Surek, "High speed EFG of wide silicon ribbon," Journal of Crystal Growth, vol. 50, no. 1, pp. 175-192, 1980.

[6] R. L. Wallace, J. I. Hanoka, A. Rohatgi, and G. Crotty, "Thin silicon string ribbon," Solar Energy Materials and Solar Cells, vol. 48, no. 1-4, pp. 179-186, 1997.

[7] J. I. Hanoka, "Overview of silicon ribbon growth technology," Solar Energy Materials and Solar Cells, vol. 65, no. 1, pp. 231-237, 2001.

[8] H. Lange and I. A. Schwirtlich, "Ribbon Growth on Substrate (RGS) - a new approach to high speed growth of silicon ribbons for photovoltaics," Journal of Crystal Growth, vol. 104, no. 1, pp. 108-112, 1990.

[9] S. N. Mohammad, M. A. Sobhan, and S. Qutubuddin, "The influence of grain boundaries on the performance efficiency of polycrystalline gallium arsenide solar cells," Solid State Electronics, vol. 32, no. 10, pp. 827-834, 1989.

[10] ASTM E112-12, Standard Test Methods for Determining Average Grain Size, ASTM International, West Conshohocken, Pa, USA, 2012.

[11] Z. Zhou, S. Mukherjee, and W.-K. Rhim, "Measurement of thermophysical properties of molten silicon using an upgraded electrostatic levitator," Journal of Crystal Growth, vol. 257, no. 3-4, pp. 350-358, 2003.

[12] M. G. Mauk, "Silicon solar cells: physical metallurgy principles," JOM, vol. 55, no. 5, pp. 38-42, 2003.

[13] L. H. Skolnik, W. P. Allred, and W. G. Spitzer, "An infrared localized vibrational mode technique for measuring segregation coefficients," Journal of Physics and Chemistry of Solids, vol. 32, no. 1, pp. 1-14, 1971.

[14] T. F. Ciszek, "Maximum growth rates for melt-grown ribbon-shaped crystals," Journal of Applied Physics, vol. 47, no. 2, article 440, 1976.

[15] J. P. Kalejs, "Point defect, carbon and oxygen complexing in polycrystalline silicon," Journal of Crystal Growth, vol. 128, no. 1-4, pp. 298-303, 1993.

[16] A. Gülhan, "Measurement techniques for high enthalpy and plasma flows," Tech. Rep. 9A-1-9A-17, RTO EN-8, The NATO Science and Technology Organization, Brussels, Belgium, 1999. 

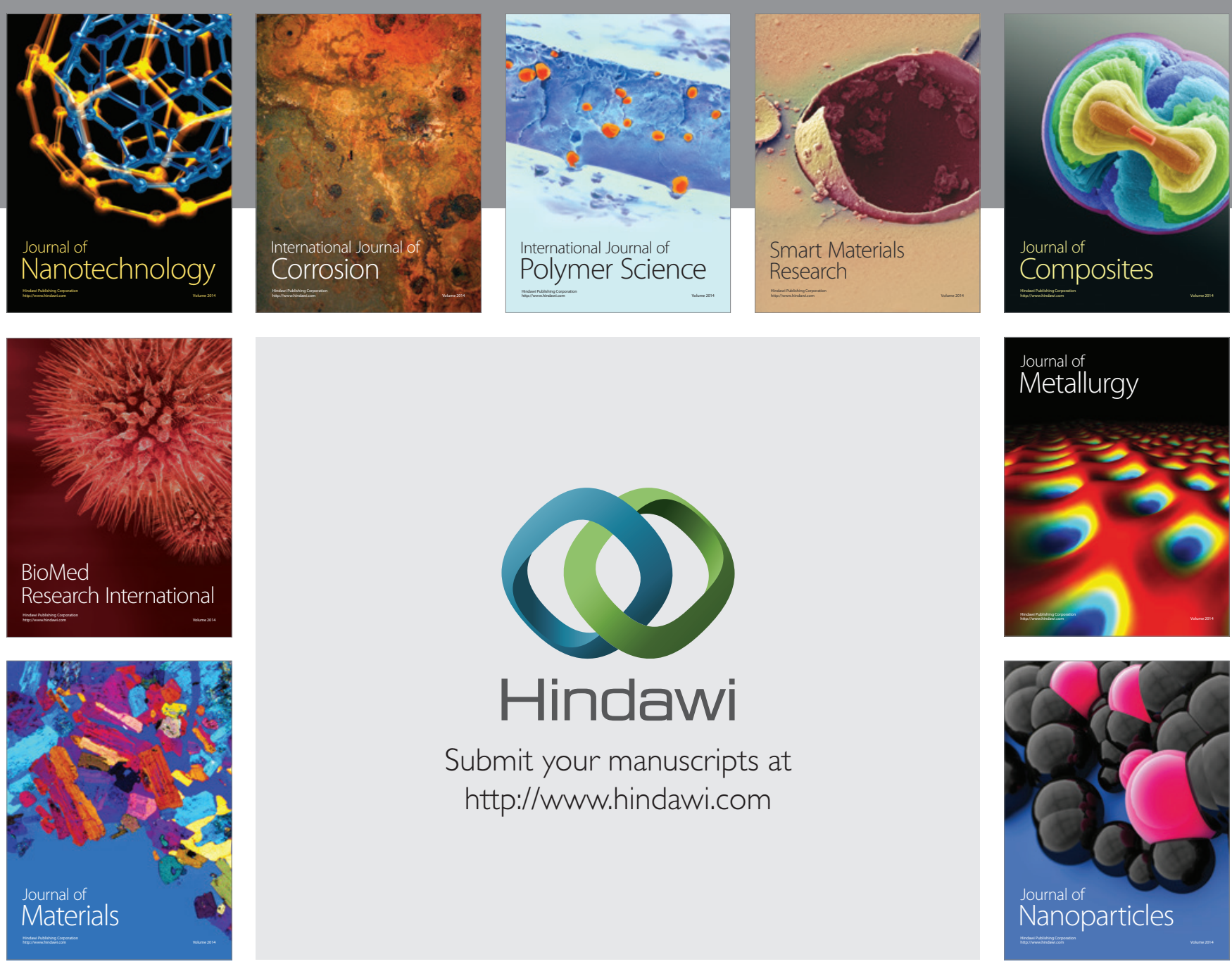

Submit your manuscripts at http://www.hindawi.com
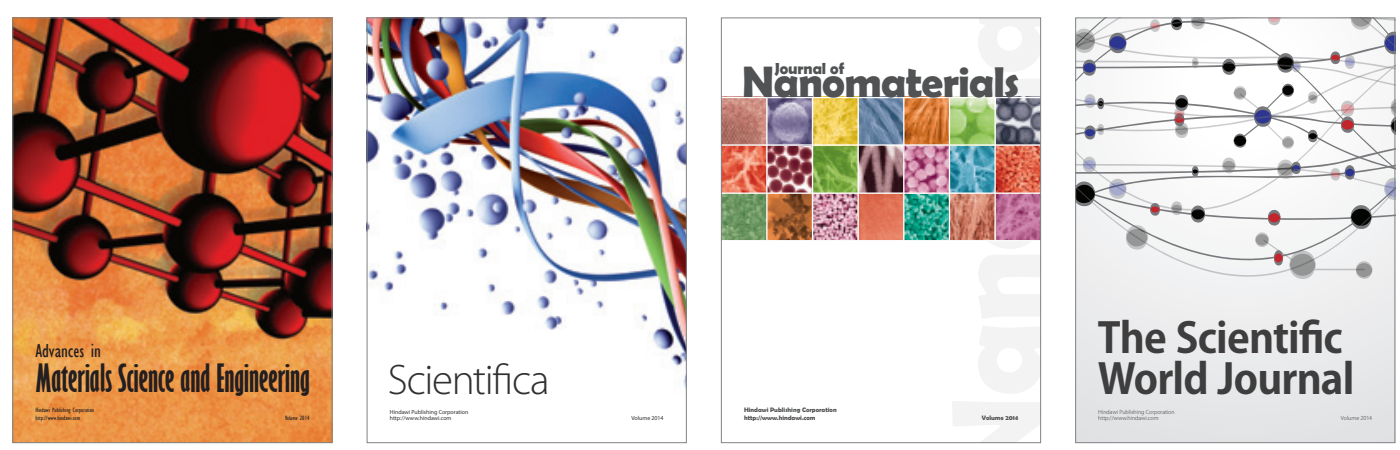

\section{The Scientific World Journal}
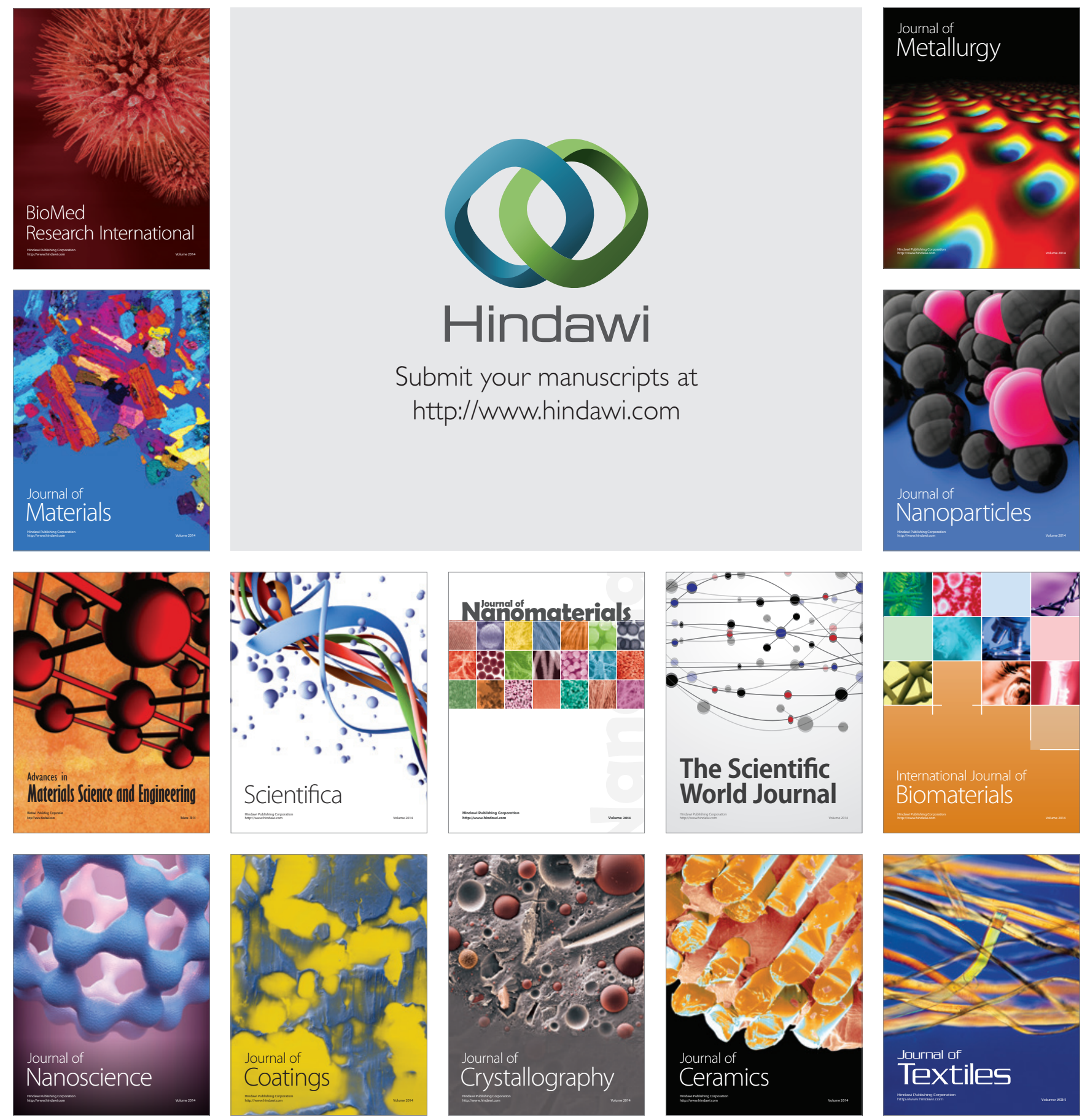